\title{
Stopping, rationalising or optimising antipsychotic drug treatment in people with
} intellectual disability and/or autism

Rohit Shankar ${ }^{1,2 *}$, Mike Wilcock ${ }^{3}$, Katy Oak ${ }^{3}$, Paula McGowan ${ }^{4}$, Rory Sheehan ${ }^{5}$

${ }^{1}$ Cornwall Partnership NHS Foundation Trust, Truro, United Kingdom

${ }^{2}$ University of Exeter Medical School, Exeter, United Kingdom

${ }^{3}$ Royal Cornwall Hospitals Trust Truro, United Kingdom

${ }^{4}$ Expert by Experience

${ }^{5}$ University College London United Kingdom

${ }^{*}$ Corresponding author

Email: Rohit.shankar@nhs.net

\section{Key Learning points}

Antipsychotic medication is often prescribed to adults with intellectual disability and/or autism to manage behaviour that challenges despite little research evidence that antipsychotics are effective

The STOMP campaign is aimed at reducing inappropriate prescribing of antipsychotic medication for people with ID and/or autism

There is an absence of robust evidence on the most effective way to reduce or stop antipsychotic medication

Withdrawing medication requires a multidisciplinary approach, consideration of co-morbidity and the involvement of patients and their carers 


\section{Introduction}

Intellectual disability (ID; also known as learning disability) is characterised by significant impairment of both cognitive functioning and adaptive behaviours, and an onset in early childhood. People with intellectual disability experience a different pattern of morbidity to the general population and die considerably younger than their counterparts without ID. ${ }^{1}$ Autism is a neurodevelopmental disorder characterised by troubles with social interaction and communication, and by restricted and repetitive behaviour. In both conditions, complex mental and physical health problems, as well as social issues, are common and are associated with communication difficulties that can result in maladaptive behaviour patterns (often referred to as 'behaviour that challenges'). Ideally, all people presenting with behaviour that challenges should be assessed by a specialist multidisciplinary team (comprising psychiatrists, psychologists, speech and language therapists, occupational therapists) to develop an understanding of the behaviour and an appropriate support plan with tailored treatment strategies and specialist follow-up. ${ }^{2}$ Non-pharmacological interventions for challenging behaviour, such as positive behaviour support or cognitive behaviour therapy, and manipulation of environmental triggers, are preferred to psychotropic medication. However antipsychotic medication is often prescribed to adults with ID and/or autism to manage behaviour that challenges in the absence of severe mental illness, despite there being little research evidence that antipsychotics are effective in this context. ${ }^{3}$

\section{Problems with antipsychotics}

There are complexities in prescribing, dispensing and administering psychotropic medication for adults with ID and/or autism, and a significant proportion will lack capacity to consent to treatment. ${ }^{4}$ There is widespread multi-morbidity and polypharmacy, increasing the potential for drug-disease and drug-drug interactions. In addition, people with ID and/or autism tend to be more sensitive to adverse drug effects and have atypical responses to drug treatment. A 
national report that highlighted concerns related to overuse of psychotropic medication in people with ID and/or autism, was followed by data from population-based studies describing the scale of psychotropic prescribing in this group of people, a national guideline on assessment and management of behaviour that challenges, a NHS quality improvement report on optimising use of medicines, and the national Stopping Overuse of Medication in People with Learning Disabilities and/or autism (STOMP) campaign. 2,5-9.

\section{Targeting antipsychotic use}

The STOMP campaign is part of the NHS England 'call to action' aimed at reducing inappropriate psychotropic prescribing for people with ID and/or autism. Although it has achieved widespread publicity and support from professional and lay groups, the programme has been limited by a lack of evidence-based advice for clinicians, patients, and carers, on an approach to antipsychotic medication reduction. The National Institute for Health and Care Excellence (NICE) guideline on interventions for people with learning disabilities whose behaviour challenges, highlighted the importance of documenting a rationale for medication, how long the medication should be taken for and a strategy for reviewing and stopping medication. ${ }^{2}$ These resources have been highlighted in Box 1.

However, there is little practical instruction about how best to achieve this. The clinical challenge is the safe and successful reduction of antipsychotic drugs used for behaviour that challenges, particularly in those who have been prescribed medication for extended periods of time. This sits within a broader approach of medication optimisation to maximise the benefit and minimise the harms of prescribed medication for the benefit of the individual.

\section{Evidence so far}


The authors of a major systematic review (21 studies, 1027 participants) concluded that antipsychotics can be reduced or discontinued in a substantial proportion of adults with ID who use them for behaviour that challenges, although not always without adverse effects. ${ }^{10}$ There were potential benefits in weight reduction, metabolic markers and cognitive function when antipsychotic drugs were withdrawn. In the 10 studies that reported success of medication reduction programmes, the proportion of participants who discontinued antipsychotics ranged from $4 \%$ to $74 \%$, the proportion maintained on a reduced antipsychotic dose at follow-up was between $19 \%$ and $83 \%$, and the proportion unsuccessful in attempts to reduce or discontinue antipsychotics was between $0 \%$ and $96 \%$. However, the overall quality of evidence was poor and studies were prone to bias. Harms associated with medication reduction included worsening of behaviour and exposure (and deterioration) of previously undiagnosed mental illness. The review authors acknowledged that antipsychotics are likely to have a role as part of the management of some cases of behaviour that challenges in adults with ID, and concluded that there remains relatively little information to guide practice in reducing or discontinuing antipsychotic use.

Despite significant attention, there has been little in the way of empirical evidence since this review. A randomised controlled trial funded by the UK National Institute for Health Research tested the outcome of a medication withdrawal programme for adults with ID and no diagnosis of psychosis who were prescribed risperidone for behaviour that challenges. ${ }^{11}$ Participants were randomised to either their usual dose of antipsychotic or gradually reducing doses over a six-month period with placebo to maintain blinding. Recruitment was difficult and slow, largely as a result of practical issues in identifying and consenting suitable participants, and carer concerns regarding re-emergence of challenging behaviour with medication reduction. The trial's organisers planned to recruit 310 participants but only 22 were randomised and the study was under-powered to detect differences between groups. The authors noted that concerns about taking part were likely to have been exacerbated by 
limited availability of alternative interventions to manage behaviour and that there is a need for focused support and alternative interventions in conjunction with medication reduction.

A recent Dutch study aimed to identify determinants of successful and unsuccessful antipsychotic discontinuation attempts in people with ID who were prescribed these medications for behaviour that challenges. ${ }^{12}$ Eligible participants were those with ID over the age of 5 years, living in specialist accommodation who were prescribed antipsychotic medication for behaviour that challenges in the absence of severe mental illness. A reduction schedule of $12.5 \%$ of baseline dose every two weeks was chosen and was completed unblinded. The primary outcome was achievement of complete discontinuation at 16 weeks. Changes in the Aberrant Behavior Checklist (ABC), a validated scale of behaviour that challenges, was a secondary outcome. Of 499 eligible people, 129 were recruited. Reasons for non-participation were clinician concern that discontinuation might increase challenging behaviours, and concurrent changes in "clients' environment". Of the 129 participants, $61 \%$ had completely discontinued antipsychotics at 16 weeks, although subsequent re-prescribing reduced the figure to $46 \%$ at 28 weeks, and $40 \%$ at 40 weeks. Total $A B C$ scores increased in almost half of participants with unsuccessful discontinuation at 16 weeks, highlighting the possible risks of embarking on a reduction programme. Autism, higher baseline dose of antipsychotic medication, and higher ABC scores were associated with a lower likelihood of complete discontinuation. Overall, $40 \%$ of participants with ID who had been judged by their responsible clinician to be eligible for a withdrawal trial of their long-term, off-label antipsychotic drug were able to discontinue over 4-7 months, with, on average, no behavioural worsening. However, selection bias limits the generalisability of these findings.

As well as individual clinical factors, setting and staff characteristics also seem to strongly influence the ability to reduce of antipsychotic drugs. There is evidence to suggest that staff and environmental variables have a significant effect on success of antipsychotic reduction 
attempts. ${ }^{13}$ Favourable environmental conditions and good support can increase the proportion of people in whom reduction attempts are successful. Such observations highlight the importance of informed commissioning decisions and building consensus with family and carers prior to initiating antipsychotic reduction.

\section{Practical issues}

The absence of robust evidence means that universal and precise instructions for reducing medication elude us, and a pragmatic and individualised approach to deprescribing antipsychotic medication used for behaviour that challenges is needed. We have argued for rational, rather than rationed, prescribing of psychotropic medications and an emphasis on medication optimisation, rather than total medication withdrawal. ${ }^{14}$ For some, this may mean reducing medication to a minimally effective dose, without reaching complete discontinuation.

A medication optimisation approach requires shared decision-making and consideration of individual and contextual factors. Understanding an individual and their concerns should form the starting point of a discussion about medication use. Obtaining a chronology to identify when and why antipsychotic medication was prescribed is recommended, although can be difficult in practice when a drug may have been prescribed for many years and people have moved between services and care providers.

The possible advantages and disadvantages of medication continuation or reduction should be explained making use of adapted communication techniques and materials, as necessary, and including family members and paid carers where appropriate. Written resources can be provided to aid decision making. Where an individual lacks capacity to make decisions about medication, a best interest decision should be made in line with the provisions of the Mental Capacity Act. It is important to communicate and record discussions 
and decisions clearly, including any challenges or barriers, particularly social and environmental ones, which prevent the partial or full reduction of antipsychotic medication. The commitment from all parties must be recognised.

Provision of alternative approaches to managing behaviour that challenges is important to instil confidence in pursuing medication change and to mitigate any behavioural worsening during dose reductions (see table 1 and pathway 1 ). This includes an up-to-date and feasible behaviour support plan, with contingency planning and options for crisis management. The optimum rate of medication reduction is not defined and should be determined individually. There is little information on how commonplace or successful it is to add an alternative psychotropic drug during the course of antipsychotic reduction. Although this may overcome the psychological barrier of being without medication, we must remember that no psychotropic class has been shown to improve behaviour that challenges in the absence of mental illness, and substituting one medication class for another may merely prolong inappropriate prescribing.

It is good practice to ensure baseline physical investigations and blood tests are completed and records on physical health updated prior to medication change. Ensuring a monitoring framework is in place is important and could be negotiated between primary and secondary care. ${ }^{15}$ This might require suitable education and discussion with primary care stakeholders. ${ }^{16}$

Where cases have proved to be challenging 'learning from experience' meetings within continued professional development networks might help to generate new ideas and share good practice.

The major pitfalls to be mindful of in reducing antipsychotic medication used for behaviour that challenges include: 


\section{Unmasking a hitherto unrecognised mental illness}

To minimise this risk it is important that all relevant stakeholders understand markers of relapse and are encouraged and supported to report any changes noticed during reduction. It might help to agree on a slow titration schedule giving 6 to 12 weeks gaps between each dose reduction. For complex cases, consideration should be given to assessing mental state and wellbeing clinically and using structured tools such as the mini-PAS-ADD (Psychiatric Assessment Schedule for Adult with Developmental Disability) and HoNOS-LD (Health of the Nation Outcome Scales for People with Learning Disabilities) at baseline and at each stage of medication reduction.

2. Misinterpreting concurrent physical, psychological or social changes Confounders in the form of physical health conditions (e.g. pain, acute infection), psychological issues (e.g. new stress, bereavement) or social issues (e.g. carer change, changes in support environment) can impact an individual's mental state. This might lead to diagnostic overshadowing and incorrectly attributing a change to the medication reduction. It is important to recognise with the individual's broader needs and, if necessary, temporarily halt or slow the rate of antipsychotic withdrawal.

\section{Inappropriate medication use}

In some cases, medication is used as a substitute for a social or psychological intervention. A full medication review should identify inappropriate use and provide justification and impetus for the withdrawal attempt. This clarity should ensure the correct framework of support exists to facilitate withdrawal.

\section{Multimorbidity leading to challenging behaviour}

There needs to be a good understanding of co-morbidities, particularly neurodevelopmental conditions (e.g. autism and attention deficit hyperactivity disorder) and genetic conditions that are associated with behaviour that challenges. Epilepsy, present in roughly $25 \%$ of people with ID and $33 \%$ of those with autism, and/or its 
treatment can be associated with behaviour that challenges. Specialist assessment may be required if there is uncertainty in this area.

\section{Future developments}

Other interventions that have been suggested to aid medication withdrawal include the use of community-based specialist behaviour therapy teams, anger management with cognitive behaviour therapy and manipulation of social, physical and personal environment (nidotherapy). ${ }^{17-19}$ However, there is insufficient evidence to allow them to be recommended.

\section{Resources}

NHS England:

- STOMP webpages - https://www.england.nhs.uk/learning-disabilities/improvinghealth/stomp/

- STOMP presentation - https://www.england.nhs.uk/publication/stopping-over-medicationof-people-with-a-learning-disability-autism-or-both/

National Institute for Health and Care Excellence:

- Guideline (NG11) - https://www.nice.org.uk/guidance/ng11

- Medication clinic proforma (shared learning example) https://www.nice.org.uk/Media/Default/sharedlearning/Audit\%20Tool\%204th\%20draft\%2 $\underline{0(4) \cdot d o c}$

\section{Conclusion}

Where antipsychotic medication review leads to a decision to reduce or discontinue medication, the focus needs to be on improving an individual's health whilst minimising the risk of harm. The risk of continuing medication also needs to be weighed against the potential harm it can cause while contributing to a good quality of life. This requires multistakeholder involvement and systems which can review decisions on a regular basis. For services in which there are a number of people who may benefit from medication reduction or discontinuation, a priority list based on assessment of complexity, potential or actual adverse effects, and the use of restrictive practice, might be useful. Patients who are on very 
low doses of antipsychotics may be perceived as prime candidates for a 'quick win'. However, as even small doses of medication can have influence on patient presentation, due consideration still needs to be given to an appropriate discontinuation and review process.

Withdrawing medication, particularly psychotropic medication, can be challenging and requires input from doctors, specialist nurses, speech and language therapists, occupational therapists, psychologists, social workers and pharmacists. It is vital to gain the confidence of the person with ID and/or autism and their family and carers before embarking on medication reduction, and to provide alternative management and support. 


\section{References}

1. Heslop $P$, Blair $P$, Fleming $P$ et al. The confidential inquiry into premature deaths of people with intellectual disabilities in the UK: a population-based study. Lancet 2014;383:889-95.

2. National Institute for Health and Care Excellence, 2015. Challenging behaviour and learning disabilities: prevention and interventions for people with learning disabilities whose behaviour challenges (NG11) [online]. Available: https://www.nice.org.uk/guidance/ng11 [Accessed 22 October 2018].

3. Matson JL, Neal D. Psychotropic medication use for challenging behaviors in persons with intellectual disabilities: an overview. Res Dev Disabil 2009;30:572-86.

4. Trollor J, Salomon C, Franklin C. Prescribing psychotropic drugs to adults with an intellectual disability. Aust Prescr 2016;39:126-30.

5. Department of Health, 2012. Transforming care: a national response to Winterbourne View Hospital [online]. Available:

https://assets.publishing.service.gov.uk/government/uploads/system/uploads/attachment da ta/file/213215/final-report.pdf [Accessed 22 October 2018].

6. Public Health England, 2015. Prescribing of psychotropic drugs to people with learning disabilities and/or autism by general practitioners in England [online]. Available: http://webarchive.nationalarchives.gov.uk/20160704150153/http://www.improvinghealthandli ves.org.uk/publications/1248/Prescribing of psychotropic medication for people with lear ning disabilities and autism [Accessed 22 October 2018].

7. Sheehan R, Hassiotis A, Walters $K$ et al. Mental illness, challenging behaviour, and psychotropic drug prescribing in people with intellectual disability: UK population based cohort study. BMJ 2015;351:h4326.

8. NHS England, 2015. Winterbourne medicines programme: improving the use of medicines in people with learning disabilities [online]. Available:

http://webarchive.nationalarchives.gov.uk/20160805132941/http:/www.nhsiq.nhs.uk/media/2 671659/nhsiq winterbourne medicines.pdf [Accessed 22 October 2018].

9. NHS England, 2018. Stopping over-medication of people with a learning disability, autism or both (STOMP) [online]. Available at: https://www.england.nhs.uk/learningdisabilities/improving-health/stomp/ [Accessed 22 October 2018]. 
10. Sheehan R, Hassiotis A. Reduction or discontinuation of antipsychotics for challenging behaviour in adults with intellectual disability: a systematic review. Lancet Psychiatry 2017;4:238-56.

11. McNamara R, Randell R, Gillespie D et al. A pilot randomised controlled trial of community-led Antipsychotic Drug Reduction for Adults with Learning Disabilities. Health Technol Assess 2017;21:1-92.

12. deKuijper GM, Hoekstra PJ. An open-label discontinuation trial of long-term, off-label antipsychotic medication in people with intellectual disability: determinants of success and failure. J Clin Pharmacol 2018;58:1418-26.

13. Ahmed Z, Fraser W, Kerr MP, et al. Reducing antipsychotic medication in people with a learning disability. Br J Psychiatry 2000;176:42-6.

14. Sheehan R, Hassiotis A, 2017. Psychotropic medication: finding ways forward for adults with intellectual disabilities [online]. Available at:

https://www.nationalelfservice.net/treatment/antipsychotics/psychotropic-medication-findingways-forward-for-adults-with-intellectual-disabilities-rsmpsychotropics/ [accessed 22 October 2018].

15. Shankar R, Bradley-Smith G, Brigham P et al. Patient- and carer-held health records: can they improve annual health checks for patients with learning disability? Br J Gen Pract 2016;66:210-2.

16. Shankar R, Wilcock M. Improving knowledge of psychotropic prescribing in people with intellectual disability in primary care PLOS One 2018;13:e0204178.

17. Hassiotis A Robotham D, Canagasabey A et al. Randomized, single-blind, controlled trial of a specialist behavior therapy team for challenging behavior in adults with intellectual disabilities. Am J Psychiatry 2009:166;1278-85.

18. Willner P Rose J, Jahoda A et al. Group-based cognitive-behavioural anger management for people with mild to moderate intellectual disabilities: cluster randomised controlled trial. Br J Psychiatry 2013:203;288-96.

19. Tyrer $P$, Tarabi SA, Bassett $P$ et al. Nidotherapy compared with enhanced care programme approach training for adults with aggressive challenging behaviour and intellectual disability (NIDABID): cluster-randomised controlled trial. J Int Disabil Res 2017; 61: 521-31. 
Pathway Key steps in antipsychotic reduction

Review medication and consider antipsychotic reduction.

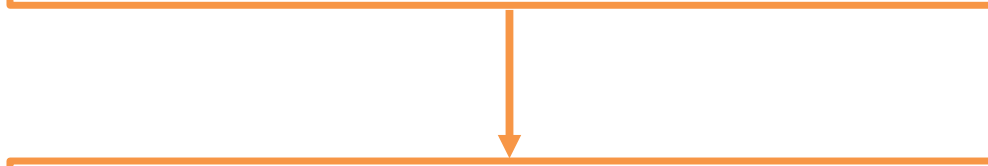

Discuss antipsychotic reduction with person with ID and carers. Explain potential benefits and risks. Aim to reach a shared decision.

Ensure alternative interventions for behaviour that challenges and input of other members of the specialist team are available before, during, and after reduction.

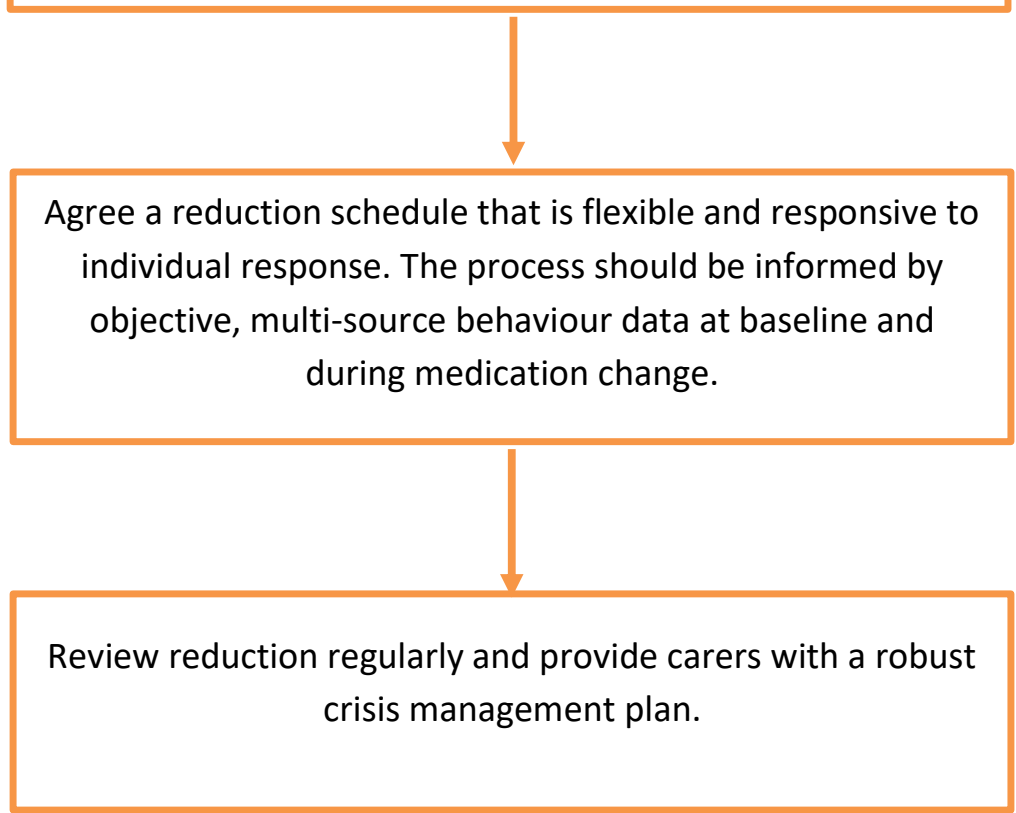


Table Good-practice principles for reducing antipsychotic medication used for behaviour that challenges in adults with intellectual disability and/or autism

For all adults who are prescribed antipsychotic drugs for behaviour that challenges

Conduct regular medication review and consider antipsychotic reduction

Medication review should form part of the learning disability annual health check, completed in primary care.

In some cases where the person with ID and/or autism is not under review by a specialist, antipsychotic reduction may be initiated by a General Practitioner who must be aware of sources of specialist support and maintain a low threshold for consultation.

\section{Before antipsychotic reduction}

Review clinical records to confirm indication for antipsychotic prescription, target

symptoms, benefits and adverse effects of antipsychotic use, and outcome of any previous discontinuation attempts.

Discuss potential risks and benefits of antipsychotic reduction with patient and carers. Aim to understand the ideas, concerns, and expectations of stakeholder groups.

Support the medication discussion with accessible information and other reasonable adjustments.

A formal best interest decision meeting may be helpful.

\section{Planning antipsychotic reduction}

Give a clear rationale for antipsychotic reduction.

Establish current baseline level of behavioural symptoms and adverse drug effects with validated measures.

Avoid major changes to antipsychotic prescription at the same time as significant changes in life circumstances (e.g. accommodation, care team, bereavement).

Ensure the programme of reduction is clear and communicated to all relevant parties (e.g.. patient, carers, family, GP, other professionals who may be involved).

Develop a plan for medication reduction taking into account of patient and carer preferences.

Ensure good support for people with ID and their carers during antipsychotic reduction including the availability of alternative methods to manage increases in behaviour that challenges.

\section{During medication reduction}

Review medication reduction and response regularly.

Aim to collect objective data from different sources and using validated measures. Carers can be asked to keep a diary of behaviour before and during antipsychotic reduction.

Be aware of potential antipsychotic withdrawal effects e.g. dyskinesias.

Allow flexibility with the pace of medication reduction and remain amenable to halting reduction or re-introducing medication.

Include psychologist or other healthcare professionals in formulating any changes in behaviour to avoid falsely misattributing change to medication reduction.

Continue to monitor physical health (in line with guidelines) while person is prescribed antipsychotic drugs.

\section{After medication reduction}

Continue to review the person and be alert for increase in behaviour that challenges or emergence of symptoms of mental illness. 